\title{
Transient regional osteoporosis presenting as a septic arthritis
}

\author{
T.E. McAlindon, S.A. Ward, D. MacFarlane ${ }^{1}$ and J.A. Mathews
}

Department of Rheumatology, St Thomas' Hospital, London SE1 7EH and 'Department of Rheumatology, Homeopathic Hospital, Tunbridge Wells, Kent TN1 IJU, UK

\begin{abstract}
Summary: A 52 year old man developed a painful swollen left knee. Clinically there was an effusion and radiographs showed local osteoporosis. Sepsis was suspected but tests proved negative and the symptoms gradually settled. During the course of the following year he developed similar pain migrating from the left foot to the right knee then to the right foot. Investigations showed a right knee effusion and 'punctate' radiographic lucencies in the patellae thought to be erosions. A bone scan demonstrated increased uptake at all four sites and sepsis was once again suspected. Biopsies and culture proved negative and a computed tomographic scan confirmed that the lucencies were due to focal osteoporosis rather than erosions. This case of transient regional osteoporosis illustrates two unusual features of this condition which resulted in diagnostic difficulty with respect to sepsis.
\end{abstract}

\section{Introduction}

Transient regional osteoporosis is a rare clinical syndrome characterized by severe pain in one or more joints accompanied by transient radiographic osteopenia in the absence of other recognizable synovial or bony abnormalities. ${ }^{1}$ Joint effusion and 'punctate' osteopenia have not been previously described in this condition.

\section{Case report}

A 52 year old male publican developed increasing pain and stiffness in his left knee in November 1990. $\mathrm{X}$-rays showed irregularity of the back of the patella suggestive of chondromalacia. The knee became swollen and the patient was referred for an orthopaedic opinion in March 1991. The patient was afebrile but his knee was exquisitely tender and a joint effusion was present. Radiography revealed osteoporosis involving the patella and lateral femoral condyle. Septic arthritis was suspected and the patient admitted for investigation and treatment. Full blood count, ESR and serum uric acid were normal, and auto-antibodies and rheumatoid factor were absent. Liver enzymes were moderately raised but hepatitis serology proved negative and liver ultrasound scan normal. ${ }^{99} \mathrm{Tc}-\mathrm{Hydroxymethyl}$ diphosphonate $\left({ }^{99} \mathrm{Tc}-\mathrm{HMDP}\right)$ scintigraphy showed

Correspondence: T.E. McAlindon, D.M., M.R.C.P. Accepted: 24 March 1993 a 'hot patella' and increased uptake in the lateral femoral condyle and tibial plateau. A gallium scan showed similar distribution of uptake, although less pronounced. At arthroscopy soft retro-patellar cartilage was seen, but biopsies were negative for acid and alcohol fast bacilli and were sterile on culture. He was treated with intravenous antibiotics and slowly improved.

In July the patient developed severe pain in the left foot. An X-ray demonstrated patchy osteoporosis suggestive of regional sympathetic dystrophy. One month later he developed pain in the right knee and in September the right foot also became involved. He became unable to walk unaided and was re-admitted for investigation. $\mathrm{X}$-rays showed punctate osteoporosis (reported as erosions) involving both patellae and femoral condyles, and a small right knee effusion (Figure 1). Full blood count and ESR were normal, C-reactive protein 13 (CRP13) immunoglubulins and serology were negative and repeat patellar biopsy was sterile. A further bone scan showed zonal uptake at all four sites (Figure 2). Computed tomography (Figure 3) confirmed focal osteoporosis and magnetic resonance imaging (MRI) of the right knee showed marked subchondral signal changes (decreased on $T_{1}$-weighted sequences). A diagnosis of transient regional osteoporosis was made. He was treated with analgesics and physiotherapy, and was given a 3 week course of calcitonin, 100 units daily. He gradually improved and by June 1992 was fully ambulant. 


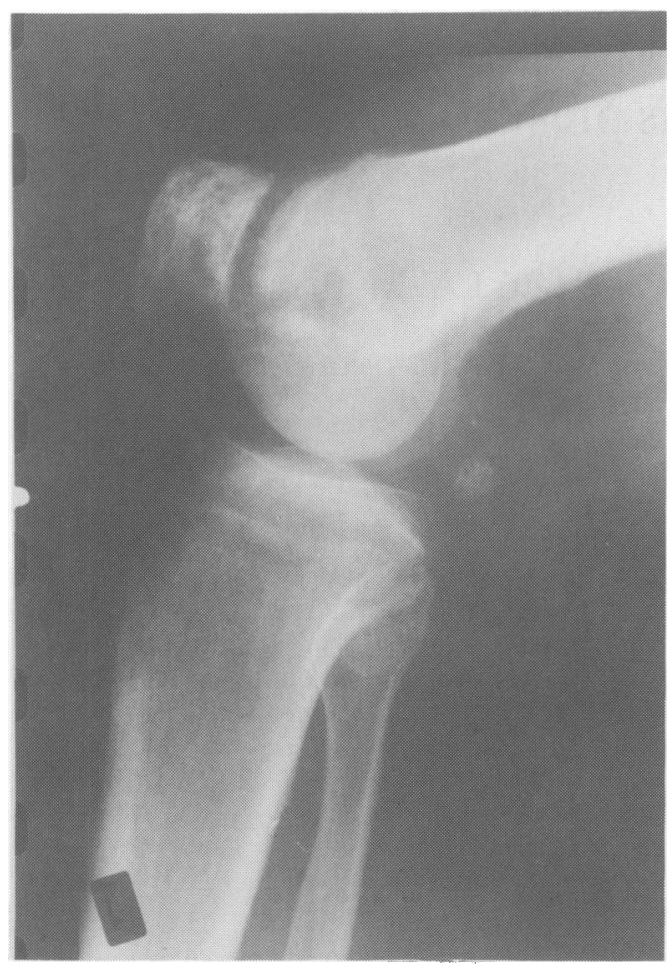

Figure 1 Lateral view radiograph of right knee showing 'punctate' osteoporosis.

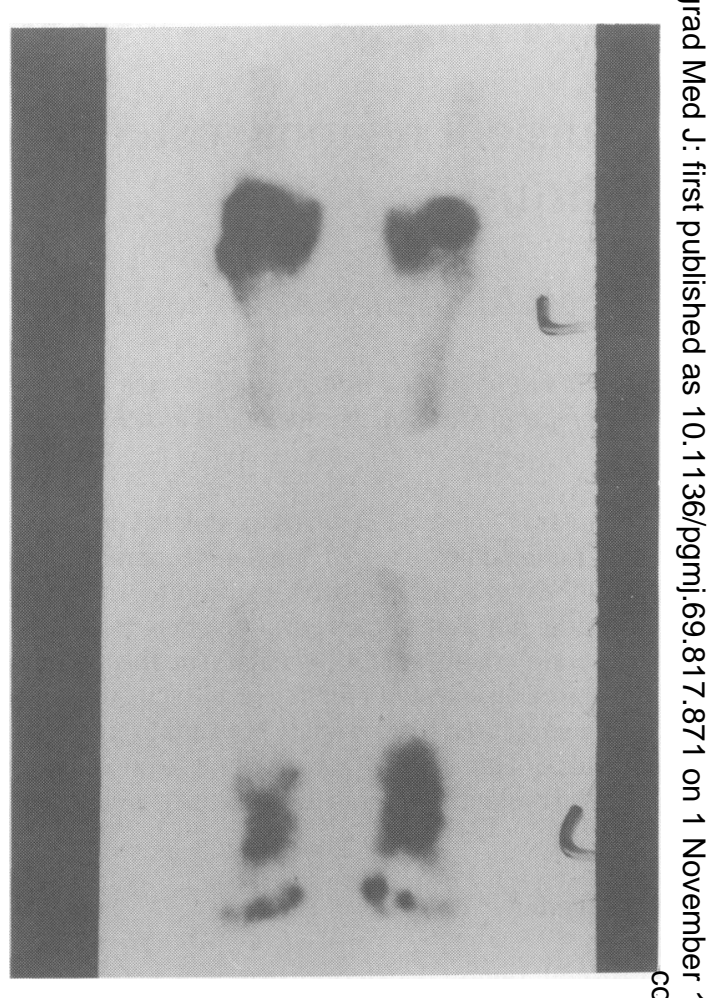

Figure $2{ }^{99}$ Tc-HMDP bone scan showing uptake knees, ankles and metatarsal phalangeal joints. के.

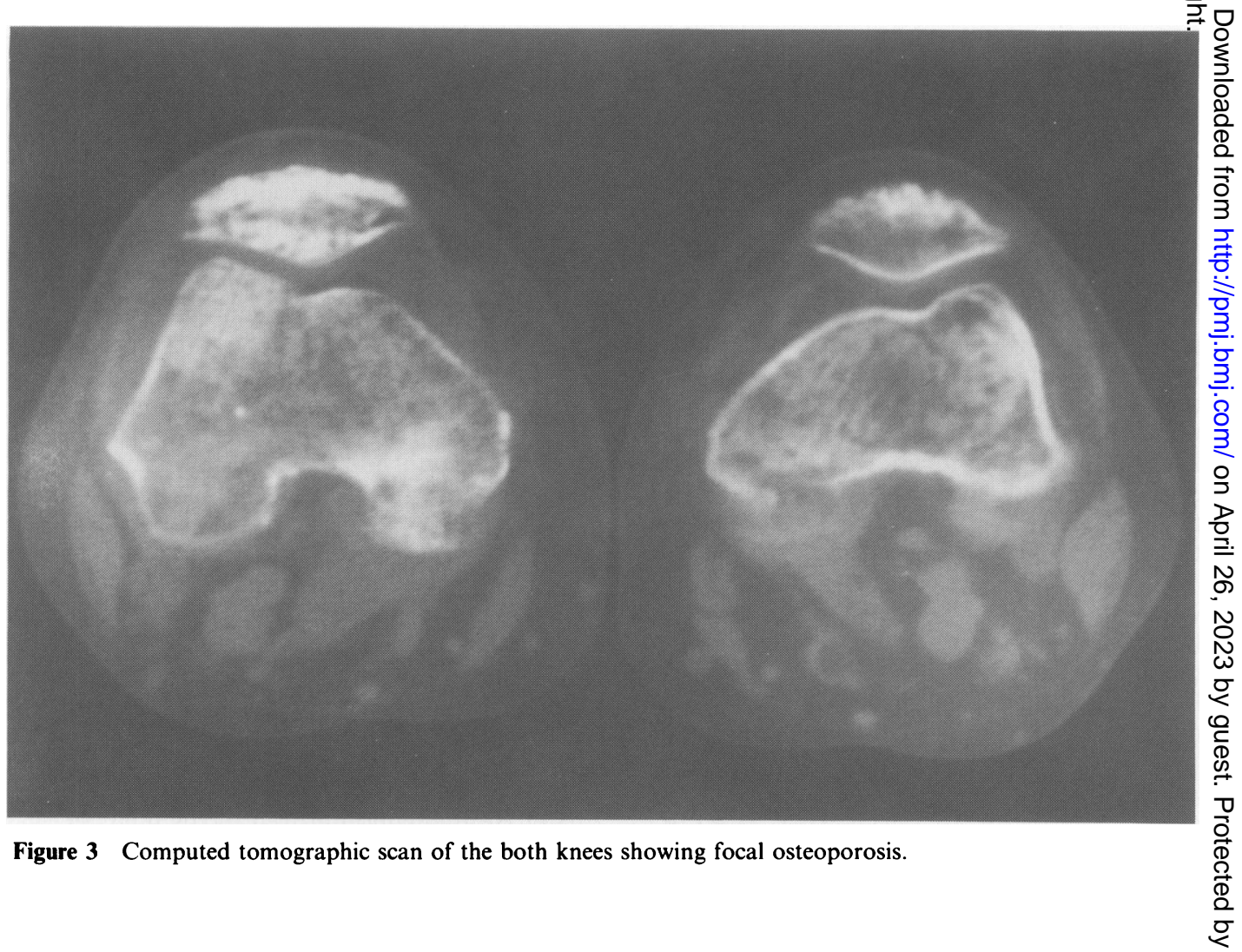




\section{Comment}

Transient regional osteoporosis is a rare condition with a propensity to afflict middle-aged men. It is thought to differ from regional sympathetic dystrophy in its tendency to involve the lower limbs, a propensity to migrate, a relative absence of neurovascular features, and a better prognosis in respect of long-term disability. ${ }^{1}$

There can be difficulty in differentiating transient regional osteoporosis from sepsis. ${ }^{2}$ Involvement of the patellae and lateral femoral condyles in this case suggested a multifocal process. Further difficulty was added by the presence of joint effusions and by the confusion of the characteristic punctate osteoporosis with erosions. That these were not erosive was clarified by computerized tomography (see Figure 3). MRI appearances

\section{References}

1. Lakhanpal, S., Ginsburg, W.W., Harvinder, L.S. \& Hunder, G.G. Transient regional osteoporosis. Ann Intern Med 1987, 106: 444-450.

2. Lequesne, M., Kerboull, M., Bensasson, M., Perez, C., Dreiser, R. \& Forest, A. Partial transient osteoporosis. Skel Radiol 1977, 2: 1-9.

3. Bloem, J.L. Transient osteoporosis of the hip: MR imaging. Radiology 1988, 167: 753-755.

4. Wilson, A.J., Murphy, W.A., Hardy, D.C. \& Totty, W.G. Transient osteoporosis: transient bone marrow edema? Radiology 1988, 167: 757-760. indicating increased marrow water content are also typical of transient osteoporosis but may be confused with avascular necrosis. ${ }^{3,4}$ The clinical course and absence of bony destruction mitigate against the latter diagnosis.

Early pointers are absence of inflammation and the presence of other 'hot' but asymptomatic joints on scintigraphy that later become painful. ${ }^{5}$

Little is known about the aetiology of transient regional osteoporosis. Transient osteoporosis of the hip, however, is a similar condition which is recorded in the third trimester of pregnancy and has been reported in a patient with cirrhosis of the liver. ${ }^{6,7}$ This male patient had abnormal liver function tests and it is possible that alcohol with its associated endocrine features may have played a role.
5. Tannenbaum, H., Esdaile, J. \& Rosenthall, L. Joint imaging in regional migratory osteoporosis. J Rheumatol 1979, 7: 237-244.

6. Coughlan, R.J., Hazleman, B.L., Crisp, A.J., Jenner, J.R. \& Page Thomas, D.P. Algodystrophy in pregnancy. Three case reports. Br J Obstet Gynaecol 1988, 95: 935-937.

7. Rozenbaum, M., Zinman, C., Nagler, A. \& Pollack, S. Transient osteoporosis of the hip joint with liver cirrhosis. $J$ Rheumatol 1984, 11: 241-243. 